\title{
Image Mosaic Algorithm Based on PCA-ORB Feature Matching
}

\author{
Juntao Zhu ${ }^{1}$ Chaofei Gong ${ }^{1, *}$ Miaoxing Zhao ${ }^{1}$ Lei Wang $^{1} \quad$ Yang Luo $^{1}$ \\ ${ }^{1}$ College of Geomatics and Geoinformation, Guilin University of Technology, \\ Guilin Guangxi 541004, China \\ ggchaofei@163.com
}

KEY WORDS: PCA-ORB, KNN, RANSAC, PCA-SIFT, Image Stitching, Image Fusion

\begin{abstract}
:
In the process of image stitching, the ORB (Oriented FAST and Rotated BRIEF) algorithm lacks the characteristics of scale invariance and high mismatch rate. A principal component invariant feature transform (PCA-ORB, Principal Component AnalysisOriented) is proposed. FAST and Rotated BRIEF) image stitching method. Firstly, the ORB algorithm is used to optimize the feature points to obtain the feature points with uniform distribution. Secondly, the principal component analysis (PCA) method can reduce the dimension of the traditional ORB feature descriptor and reduce the complexity of the feature point descriptor data. Thirdly, KNN (K-Nearest Neighbor) is used, and the k-nearest neighbor algorithm performs roughly matching on the feature points after dimensionality reduction. Then the random matching consistency algorithm (RANSAC, Random Sample Consensus) is used to remove the mismatched points. Finally, the fading and fading fusion algorithm is used to fuse the images. In 8 sets of simulation experiments, the image stitching speed is improved relative to the PCA-SIFT algorithm. The experimental results show that the proposed algorithm improves the image stitching speed under the premise of ensuring the quality of stitching, and can play a role in fast, real-time and large-scale applications, which are conducive to image fusion.
\end{abstract}

\section{PREFACE}

Image stitching has been widely studied and discussed in the field of image processing and computer vision. Its main purpose is tantamount to fuse two or more images with a certain degree of overlap into a high-resolution image with a wide field of view through image stitching technology (Zeng et al., 2018).

In 2004, Lowe (Lowe, 2004) improved the SIFT algorithm, and explained the location of the feature points and the distribution of the feature points in detail, and made corresponding experimental verification. The experimental results show that the feature points detected by the SIFT feature detection algorithm are excellent for image rotation, affine transformation, noise and illumination. In 2006, Bay (Bay et al., 2006) and others proposed an accelerated and robust feature detection algorithm on the ECCV, which is the SURF feature extraction algorithm. Compared with the SIFT algorithm, the algorithm is more stable and efficient, and the algorithm's operation speed has also been steadily improved. In 2011, Rublee (Rublee et al., 2011) and others proposed a fast feature point extraction and description algorithm: ORB algorithm. The speed of the algorithm is better than the SIFT algorithm and SURF algorithm in terms of computational efficiency and feature point extraction efficiency. Since then, the ORB algorithm (Fan et al., 2019; 2019; Lu et al., 2019; Vinay et al., 2018) has been greatly developed. On this basis, this paper adds the PCA algorithm to improve the operation speed of the ORB algorithm and improve the image stitching speed.

This paper proposes a PCA-ORB image mosaic algorithm to improve the speed and accuracy of ORB image stitching. Firstly, the algorithm uses PCA-ORB to reduce the dimension

\footnotetext{
*Corresponding author
} 
of feature descriptors to obtain a more uniform distribution of feature points, which speeds up the construction of descriptors. At the same time, KNN algorithm is used to roughly match the feature points after dimension reduction, and then adopt The RANSAC algorithm eliminates the mismatched points, and finally uses the fade-in and fade-out fusion algorithm to fuse the images.

\section{PCA-ORB ALGORITHM}

\subsection{ORB Algorithm}

The ORB feature point detection algorithm was proposed by Rublee in ICCV in 2011. The feature point detection algorithm is based on the FAST (Features from Accelerated Segment Test) algorithm and the BRIEF (Binary Robust Independent Elementary Features) algorithm. A new algorithm, the algorithm has proved to be a good feature point detection algorithm in the actual application process.

2.1.1 FAST Algorithm: The FAST algorithm was proposed by Rosten (Rosten et al., 2006) in 2006 and was revised in 2010. The algorithm is just a feature point detection algorithm, but the feature description processing cannot be implemented. The algorithm determines whether it is a feature point by comparing the pixel gray level around a candidate pixel. The main algorithm is described in the literature (Rosten et al., 2006; Liu et al., 2017). However, the feature points detected by the FAST algorithm have no direction. To compensate for this defect, the FAST feature points need to be added with direction information. Therefore, the ORB algorithm uses the oFAST algorithm. The basic idea: assuming that the intensity of a corner point deviates from its centroid, we represent this deviation distance as a vector defined as the formula (1):

$$
m_{p q}=\sum_{x, y} x^{p} y^{q} I(x, y)
$$

Where, $I(x, y)$ represents the gray value at point $(x, y), \mathrm{p}$ and $\mathrm{q}$ are the medians of this matrix. So, the centroid coordinate can be expressed as:

$$
C=\left(\frac{m_{10}}{m_{00}}, \frac{m_{01}}{m_{00}}\right)
$$

Where, $m_{10} / m_{00}$ represents the centroid of the X-axis direction, $m_{01} / m_{00}$ represents the centroid of the Y-axis direction. Taking feature point $O$ as the starting point and centroid $C$ at the end point as vector $\overrightarrow{O C}$, the direction of the vector can be used as the direction of the FAST feature point. The expression is as follows:

$$
\alpha=\arctan 2\left(m_{01}, m_{10}\right)
$$

2.1.2 The BRIEF Algorithm: The BRIEF algorithm is a binary string-based descriptor that is simple and fast to calculate. The rBRIEF (rotated BRIEF) algorithm is an improvement of the BRIEF algorithm to solve its rotation invariance. The main algorithm is described in the literature (Zhou et al., 2015; Li et al., 2017).

In the pixel area around $31 \times 31$ feature point, select a sub-window of $5 \times 5$ and find the sum of the gray values of the sub-window. The expression of the gray value is as follows:

$$
\tau(p ; x, y):= \begin{cases}1 & p(x)<p(y) \\ 0 & p(x) \geq p(y)\end{cases}
$$

Where, $p(x)$ and $p(y)$ are the sum of the gray values of two sub-windows respectively. The BRIEF feature descriptor is recorded as a binary test vector containing $\mathrm{n}$ dimensions, forming a descriptor of the feature point. The expression is as follows:

$$
f_{n}(p)=\sum_{1 \leq i \leq n} 2^{i-1} \tau\left(p ; x_{i}, y_{i}\right)
$$

Where $\mathrm{n}$ is 256 . For $\left(x_{i}, y_{i}\right)$ at any position, $\mathrm{n}$-dimensional binary test results in a $2 \times n$ dimensional matrix:

$$
S=\left[\begin{array}{llll}
x_{1} & x_{2} & \ldots & x_{n} \\
y_{1} & y_{2} & \ldots & y_{n}
\end{array}\right]
$$

The corner point direction is known by the FAST algorithm, and as showed by the formula (3), the rotation matrix is obtained as $\boldsymbol{R}_{\sigma}$ 


$$
\begin{aligned}
& R_{\alpha}=\left[\begin{array}{cc}
\cos \alpha & \sin \alpha \\
-\sin \alpha & \cos \alpha
\end{array}\right] \\
& S_{\alpha}=R_{\alpha} . S
\end{aligned}
$$

Finally, the descriptor is rBRIEF:

$$
g_{n}(p, \alpha):=f_{n}(p) \mid\left(x_{i}, y_{i}\right) \in S_{\alpha}
$$

\subsection{PCA Algorithm}

The idea of PCA algorithm is to map n-dimensional features to $\mathrm{k}$-dimensional $(\mathrm{k}<\mathrm{n})$, which is a different orthogonal feature. We call this k-dimensional feature a principal component and a reconstructed k-dimensional feature. Rather than simply removing the remaining $\mathrm{n}-\mathrm{k}$ dimension features from the n-dimensional features. It reduces feature counts, reduces noise and redundancy, and reduces the likelihood of over fitting. The main algorithm is referenced in the literature (Vinay et al., 2015; Jiang et al., 2016).

With a data matrix of $\mathrm{n}$ samples, which is $X=\left(x_{1}, x_{2}, \cdots, x_{n}\right)$, then the mean vector of these $\mathrm{n}$ samples is $\vec{x}$,

$$
\vec{x}=\frac{1}{n} \sum_{i=1}^{n} x_{i}
$$

Calculate the covariance matrix $\mathrm{S}$ of the above formula:

$$
S=\frac{1}{n} \sum_{i=1}^{n}\left(x_{i}-\vec{x}\right)\left(x_{i}-\vec{x}\right)^{T}
$$

The eigenvalues of the covariance matrix $\mathrm{S}$ is obtained, and the eigenvalues are sorted in descending order, and the largest $\mathrm{k}$ of them are selected, and then the corresponding k eigenvectors is respectively used as column vectors to form an eigenvector matrix.

The corresponding feature vector is:

$$
\varphi_{1}, \varphi_{2}, \cdots, \varphi_{k}, \varphi_{j}
$$

Where, $j=1,2, \cdots, k$. The corresponding matrix is:

$$
\phi_{K}=\left(\varphi_{1}, \varphi_{2}, \cdots, \varphi_{k}\right)
$$

So, for the sample $x$ in any n-dimensional space, there is a matrix:

$$
Z=\phi_{k}^{T}\left(x_{i}-\vec{x}\right)^{T}
$$

So, the matrix $\mathrm{Z}$ is the result of the dimensional reduction of the matrix $\mathrm{X}$

\section{IMAGE MATCHING AND FUSION}

\subsection{Feature Point Rough Matching}

After generating the feature descriptors of the two graphs, the two graphs need to be feature point matched. This paper uses the $\mathrm{K}$ nearest neighbor algorithm, also known as $\mathrm{KNN}$ (K-Nearest-Neighbor) algorithm. The algorithm is a simple algorithm for data classification. The core idea is that most samples of the most neighboring samples in the feature space belong to a certain category, and the sample also belongs to this category. In plain terms, the classification principle of the algorithm is the principle that the minority obeys the majority (Lu et al., 2017). Then use the LSH (Locality-Sensitive Hashing) search algorithm to quickly find the matching point.

\subsection{Elimination of Mismatch Points}

Feature point matching is performed by the feature point matching method described above, and there are still unavoidable mismatch points in the matching result. In order to ensure the accuracy of image matching, it is necessary to use corresponding processing methods to eliminate these mismatch points. In this paper, random sampling consistency (RANSAC) (Zhang et al., 2016) algorithm is used to eliminate the mismatched points. The RANSAC algorithm is a robust algorithm for transformation estimation. It optimizes the parametric model to eliminate the data of the "inside point". Unreasonable "outside points" to improve the correct rate of matching. The central idea is: randomly select 4 pairs of non-collinear matching points, solve the transformation matrix $\mathrm{H}$; calculate the projection error of the transformation matrix $\mathrm{H}$ and the matching point set and set a threshold. If the error is less than the threshold, it is recorded as the inner point. On the contrary, it is recorded as an external point; all the outer points are eliminated by multiple repeated selections.

\subsection{Image Fusion}

After the image is registered, the spatial relationship between the images has been determined. At this time, the fusion algorithm has to be selected. In the selection of the fusion 
algorithm, we should pay attention to the following two key elements: one is to carry out the fusion operation only for the overlapping area and try to keep the original image information unchanged; the other is to maintain the smooth transition of the fusion boundary, which can effectively avoid the spelling generated during the splicing (Yang et al., 2002). So, this paper uses the gradual involution fusion algorithm proposed by Szeliski (Szeliski, 1996).

The specific fusion algorithm is as follows:

$$
f(x, y)=\left\{\begin{array}{cc}
M(x, y) & (x, y) \in M \\
P_{1} * I(x, y)+P_{2} * J(x, y) & (x, y) \in(I \cap J) \\
N(x, y) & (x, y) \in N
\end{array}\right.
$$

In the above equation, $f(x, y)$ represents the fused image, $P_{1}$ and $P_{2}$ represent the corresponding pixel weight of the image $M(x, y)$ and $N(x, y)$ in the overlapping region respectively, meeting the requirements of $P_{1}+P_{2}=1$ and
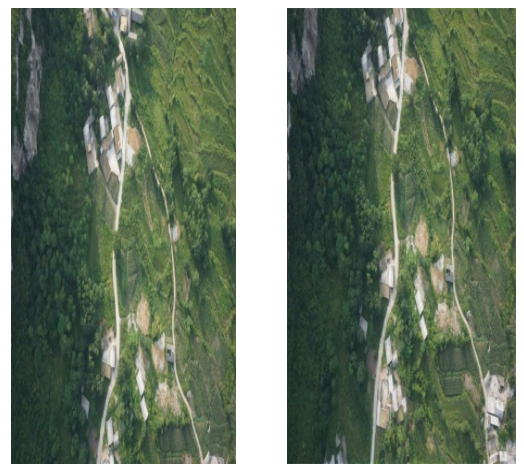

a1

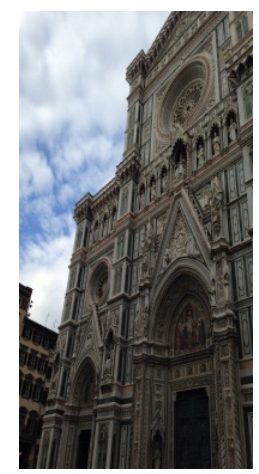

b1
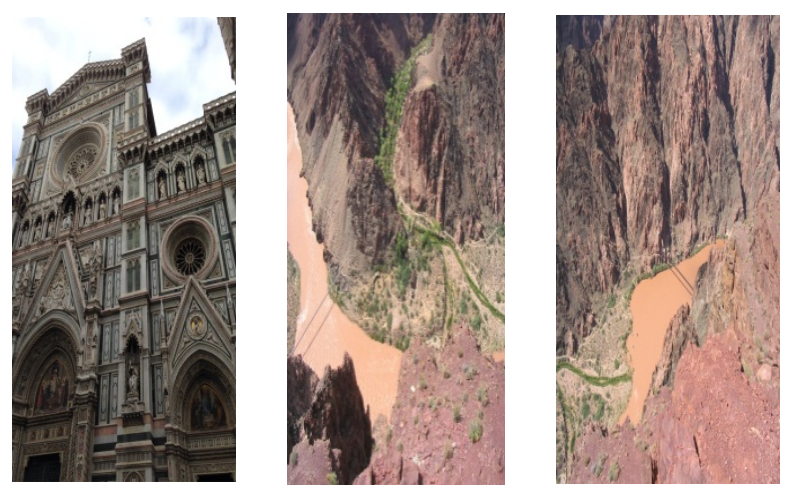

$\mathrm{c} 1$

Figure 1. Images to be stitched

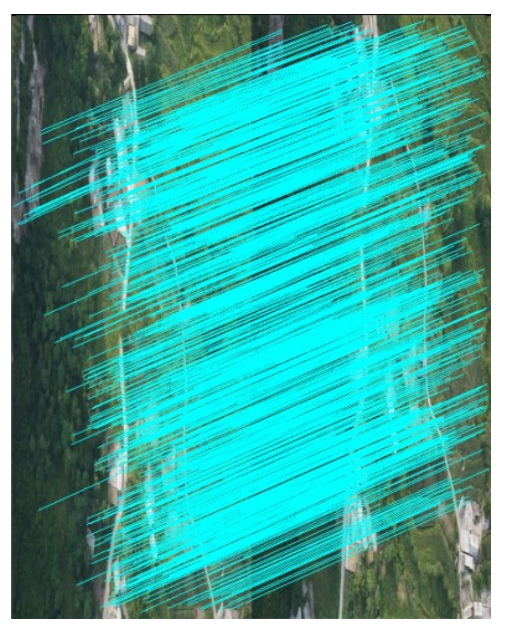

a2

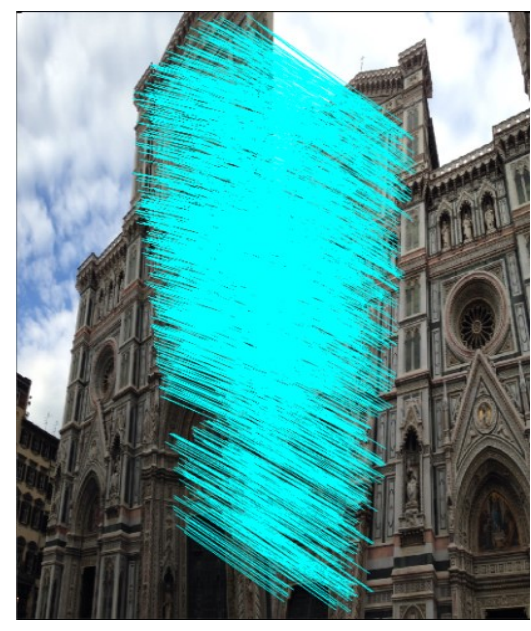

b2

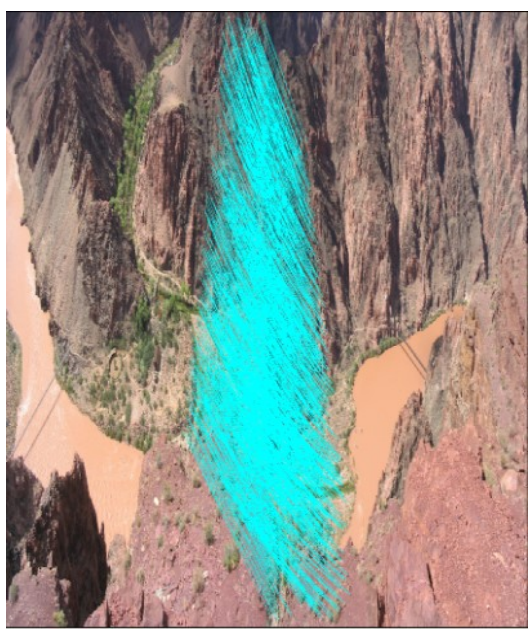

c2

Figure 2. PCA-SIFT algorithm matching results 


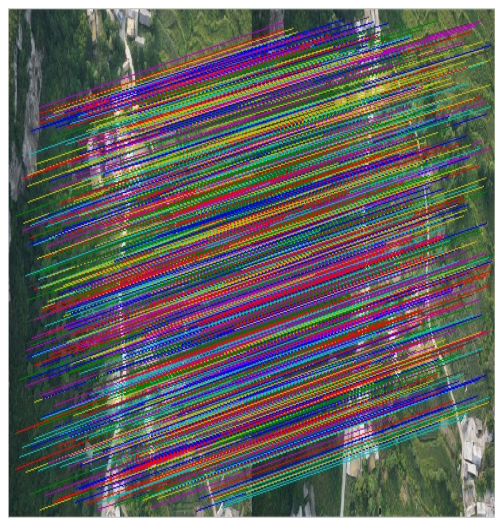

a3

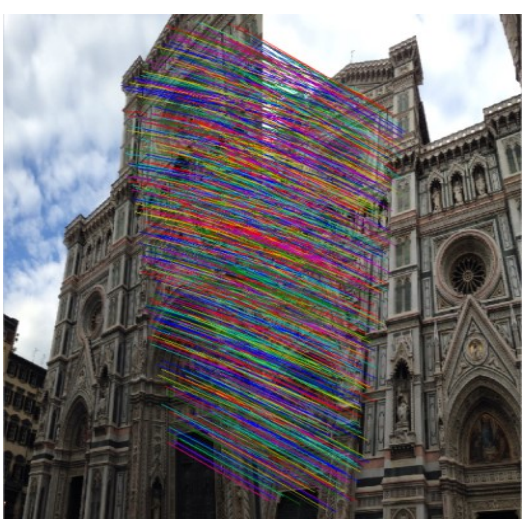

b3

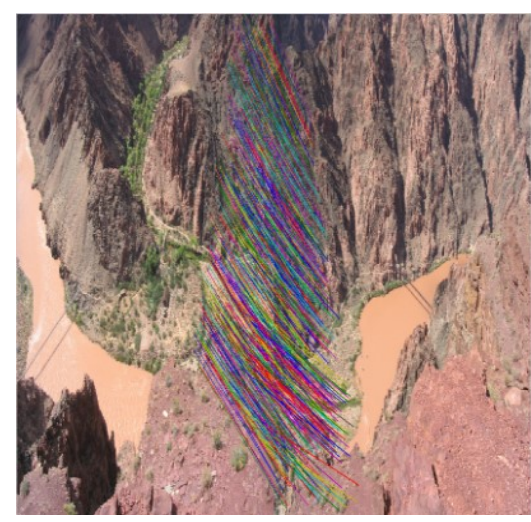

c3

Figure 3. PCA-ORB algorithm matching results

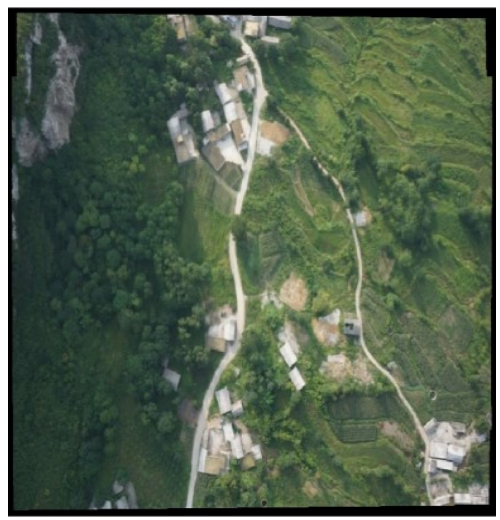

a4

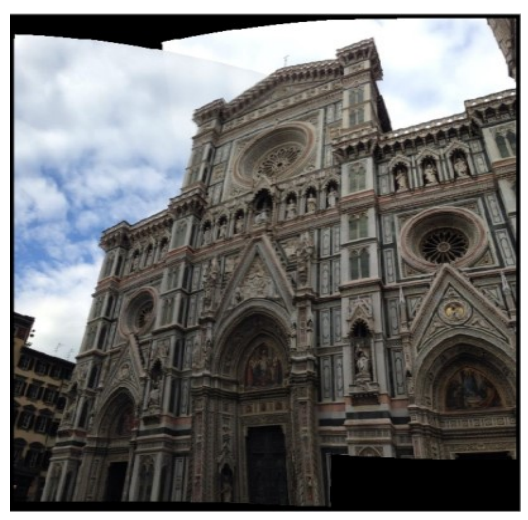

b4

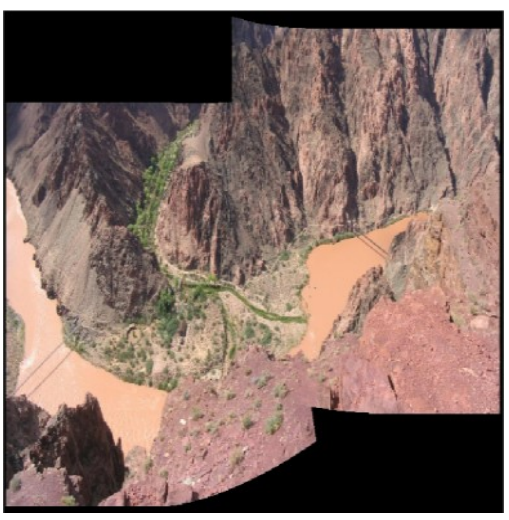

$\mathrm{c} 4$

Figure 4. PCA-ORB algorithm stitching results

In the case where the parameters are the same ones, the three groups of images in Figure 1 are spliced using the PCA-SIFT algorithm, and compared with the PCA-ORB algorithm proposed in this paper. The specific matching results are shown in Figure 2 and Figure 3. As can be seen from Figure 4, the grading of the image can be efficiently performed using the fade-in and fade-out fusion algorithm. Details of image stitching are shown in Table 1:

\begin{tabular}{ccccc}
\hline Numbering & method & $\begin{array}{c}\text { Matching } \\
\text { logarithm }\end{array}$ & $\begin{array}{c}\text { Matching } \\
\text { rate } / \%\end{array}$ & $\begin{array}{c}\text { Splicing } \\
\text { time /s }\end{array}$ \\
\hline \multirow{2}{*}{ a1 } & PCA-SIFT & 1210 & 90.12 & 14.15 \\
& PCA-ORB & 967 & 94.97 & 8.57 \\
\multirow{2}{*}{ b1 } & PCA-SIFT & 2120 & 88.34 & 21.80 \\
& PCA-ORB & 1069 & 90.38 & 10.89 \\
& PCA-SIFT & 1361 & 90.18 & 15.41 \\
& PCA-ORB & 580 & 93.65 & 4.51 \\
\hline
\end{tabular}

Table 1. Comparison of two algorithms
Among, Matching rate $=$ Matching logarithm $/$ Total matching logarithm(Yang et al., 2018).

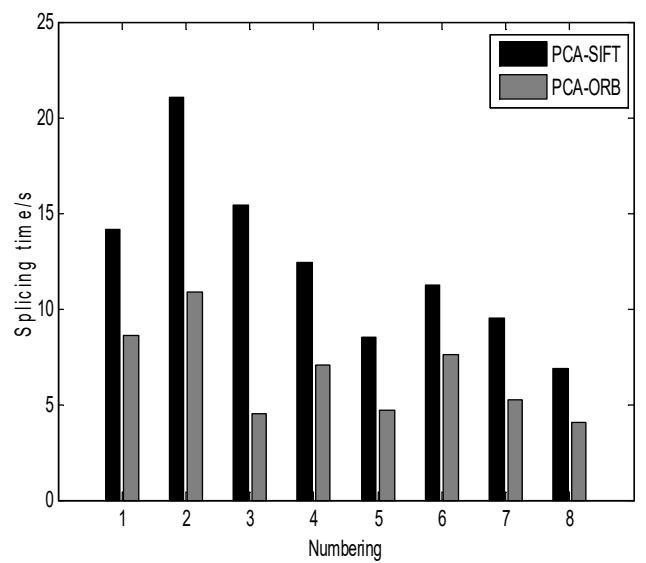

Figure 5. Images stitching time

It can be seen from Table 1 that the matching logarithm of the algorithm is reduced compared with the PCA-SIFT algorithm, but the more stable matching pairs are retained, and the 
matching rate is higher. It can be observed in Figure 5 that the image splicing time consumption of the algorithm (PCA-ORB algorithm) is less than that of the PCA-SIFT algorithm. Comprehensive Table 1 and Figure 5 can be concluded that the PCA-ORB algorithm has great advantages in feature point matching, which is conducive to image stitching.

\section{CONCLUSION}

This paper proposes an image stitching algorithm based on PCA-ORB feature matching, which can solve the problems of large data volume, high mismatch rate and a long time in the stitching process. The algorithm combines the advantages of ORB algorithm and PCA algorithm, and combines them into a new algorithm, which greatly improves the splicing speed. The experimental results show that the proposed algorithm firstly reduces the dimension of the feature descriptor, and uses the KNN algorithm to coarsely match the feature points. Then, the robust matching RANSAC algorithm is used to eliminate the mismatched points. Finally, the fading is performed using the fading inversion algorithm. The resulting image is fused. Under the premise of ensuring the quality of splicing, the algorithm is less time-consuming than the PCA-SIFT algorithm, and it is a reliable image splicing algorithm.

\section{ACKNOWLEDGMENTS}

This work was supported by National Natural Science Foundation (41461089).

\section{REFERENCES}

Bay, H., Tuyteplaars, T., Gool, L.V., 2006. SURF: Speeded Up Robust Features. Proceedings of the European Conference on Computer Version (ECCV 2006), 404-417.

Fan, X.N., Gu, Y.F., Ni, J.J., 2019. Application of Improved ORB Algorithm in Image Matching. Computer and Modernization, (2), 1-6,14.

Fan, Y.G., Chai, J.L., Xu, M.M., Wang, B., Hou, Q.S., 2019. Improved image registration based on ORB and RANSAC fusion. Optics and Precision Engineering, 27(3), 702-717.

Jiang, B., Yan, X.P., 2016. Image stitching algorithm based on PCA-SIFT feature matching. Journal of Computer Applications,36(S2),143-145.

Lowe, D., 2004. Distinctive image features from scale invariant key points. International journal of computer vision, 60(2), 91-110.

Lu, J., He, Y.Z., Chen, X., Liu, T., 2019. Improvement of ORB algorithm coupled with scale invariant features. Measurement and Control Technology, 38(3), 97-101+107.

Liu, L., Wang, P., Sun, L., 2017. Adaptive FAST Corner Detection Algorithm Based on Regional Gray Level Change. Microelectronics \& Computer, 34(3),20-24.

Li, L., 2017. Target matching algorithm is based on the image structure and improved Brief detection operator. Foreign Electronic Measurement Technology, 36(2), 29-33.

Lu, D.L., Ning, W., Yan, J., 2017. Improved KNN algorithm based on BP neural network decision making. Journal of Computer Applications, 37(2), 65-67+88.

Rublee, E., Rabaud, V., Konolige, K., 2011. ORB: an efficient alternative to SIFT or SURF. Computer Vision (ICCV), IEEE, 2564- 2571

Rosten, E., Drummond, T., 2006. Machine learning for high-speed corner detection. European Conference on Computer Vision. Springer-Verlag, 430-443.

Szeliski, R., 1996. Video mosaics for virtual environment. IEEE Computer Graphics and Applications, 16(2), 22-30.

Vinay, A., Ajaykumar, A., Cholin, S., Natarajan, S., 2018. An Efficient ORB based Face Recognition framework for Human-Robot Interaction. Procedia Computer Science, 913-923

Vinay, A., Akshay, C.K., Gaurav, R.S., 2015. ORB-PCA Based Feature Extraction Technique for Face Recognition. Procedia Computer Science, 614-621. 
Yang, F.L., Guo, H.Y., Yang, S., 2002. Research on Evaluation Method of Pixel Level Image Fusion Effect. Journal of Test and Measurement Technology,16(4),276-279.

Yang, B.K., Cheng, S.Y., Zheng, Y.Y., 2018. An Improved PCA-SIFT Algorithm for Image Mosaic. Microelectronics \& Computer, 35(12),70-75.

Zeng, H.C., Ma, Y.P., 2018. Aerial image mosaic algorithm of drone based on improving ORB. Industrial Control Computer, 31(5), 90-92.

Zhou, L.L., Jiang, F., 2015. Image Matching Algorithm Based on FAST and BRIEF. Computer Engineering and Design, 36(5), 1269-1273.

Zhang, Z., Kuang, L., Han, X., 2014. An improved RANSAC algorithm for image Mosaic. Computer Measurement \& Control, 22(6), 1856-1858. 